\title{
Independent clusters in coordinate space: an efficient alternative to shell-model expansions
}

\author{
R F Bishop†, E Buendíał, M F Flynn§ and R Guardiola§ \\ † Department of Mathematics, UMIST, PO Box 88, Manchester M60 1QD, UK \\ ¥ Departamento de Fissica Moderna, Universidad de Granada, 18071, Spain \\ § Departamento de Física Nuclear, Universidad de Valenica, 08071, Spain
}

Received 22 October 1990

\begin{abstract}
A previous shell-model-style calculation for the ground-state energy of the ${ }^{4} \mathrm{He}$ nucleus, based on coupled cluster techniques, was able to treat exactly the centre-of-mass motion. It is now recast in a precisely equivalent but vastly more computationally efficient form, directly in terms of coordinate-space correlation functions which are expanded in a Gaussian geminal basis and determined variationally. This reformulation further leads in a straightforward manner to a natural procedure for including higher-order correlations. Its implementation at even the simplest level produces a significant improvement in the already very good upper bounds achieved for the ground-state energy. Further extensions are also discussed.
\end{abstract}

In a recent letter to this journal we presented a shell-model-style calculation of the ground and $J^{+}=0$ excited states of the ${ }^{4} \mathrm{He}$ nucleus described by Wigner (central) forces. We worked in a basis generated from the non-interacting harmonic-oscillator ground state by the inclusion of all one- and two-body excitations which leave the centre-of-mass (сM) undisturbed, i.e. in the 0s state. Restricting the allowed excitations to this subset has two important consequences. Besides rendering the needed $\mathrm{CM}$ correction to the energy trivial, it drastically reduces both the number of independent basis states needed in order to include all two-particle excitations up to a given excitation energy, $n \hbar \omega$, as well as the number of harmonic oscillator states required in the intermediate sums. This latter reduction is made possible by eliminating the spurious repetitions of the calculated energy spectrum which would be produced for each allowed excitation of the $\mathrm{cm}$.

In [1] we applied our formalism to two simple effective interactions, namely the Wigner parts of the B1 potential of Brink and Boeker [2] and the V7 potential of Volkov [3], both of which are expressed analytically as a sum of Gaussians. The simplicity of our basis allowed us to include excitations of up to $60 \hbar \omega$, much higher than similar (unconverged) shell-model calculations using an unrestricted basis $[4,5]$, and we thus obtained significantly better ground-state energies. It was found that very large bases are in fact required, with convergence only being achieved for these two potentials at roughly the $40 \hbar \omega$ and $20 \hbar \omega$ level, respectively. In a later work [6], we extended the applications to two quasi-realistic microscopic interactions (since this is, after all, the type of interaction which one should be using in any attempt at a complete and converged calculation, as opposed to one restricted 
to a chosen model space). These were the spin-averaged form of the $S 3$ potential of Afnan and Tang (which is again analytically expressed as a sum of Gaussians) [7], and the MT-V potential of Malfliet and Tjon (which is expressed analytically as a sum of Yukawians) [8]. These require considerably larger basis sizes, with good convergence (i.e. at the level of a few hundredths of $1 \mathrm{MeV}$ ) for the S3 potential not being reached until the $66 \hbar \omega$ level! For the MT-V potential, convergence is still far from being achieved even at $58 \hbar \omega$ excitation energy, and larger basis sizes are not computationally practicable in this case [6]. These very poor convergence properties have led us to reconsider the entire approach and have produced the new but completely equivalent coordinate-space method described below. As we shall see, it is vastly more efficient.

The starting point for the old and the new approaches is the construction of the correlated ${ }^{4} \mathrm{He}$ ground-state wavefunction from particular combinations of correlation operators acting upon some suitable uncorrelated model state, in the usual spirit of the coupled cluster $(\mathrm{Cc})$ theory $[9,10]$. In view of our present self-imposed restriction to internucieon forces of the Wigner type (largely for pedagogical reasons, so that we may ignore from the outset the purely technical complications of spin and isospin), we may restrict our discussion to spatially symmetric wavefunctions. An obvious choice for the uncorrelated ${ }^{4} \mathrm{He}$ ground state is thus the non-interacting $(0 \mathrm{~s})^{4}$ configuration,

$$
\begin{aligned}
& \left\langle r_{1} r_{2} r_{3} r_{4} \mid \Phi_{0}\right\rangle=\prod_{i=1}^{4} \phi_{0}\left(r_{i}\right) \\
& \phi_{0}(r)=\left(\frac{\alpha^{6}}{\pi^{3}}\right)^{1 / 4} \exp \left(-\frac{1}{2} \alpha^{2} r^{2}\right)
\end{aligned}
$$

in a harmonic oscillator (Ho) potential with natural frequency $\omega=\hbar \alpha^{2} / M$, with $M$ the nucleon mass.

An arbitrary admixture of the $1 \mathrm{p}-1 \mathrm{~h}$ (one-particle-one-hole) and $2 \mathrm{p}-2 \mathrm{~h}$ states may then be created by acting on $\left|\Phi_{0}\right\rangle$ with the most general two-particle cluster correlation operator,

$$
\hat{S}_{2}=\sum_{i, j} S_{i j} a_{i}^{\dagger} a_{j}^{\dagger} a_{0} a_{0}
$$

where the sets of operators $\left\{a_{i}^{\dagger}\right\}$ and $\left\{a_{i}\right\}$ are respectively the complete sets of creation and destruction operators with respect to the vacuum state, $|\mathrm{vac}\rangle$, of our chosen Ho single-particle basis. Thus, we have

and

$$
\left|\Phi_{0}\right\rangle=(4 !)^{-1 / 2}\left(a_{0}^{\dagger}\right)^{4}|\mathrm{vac}\rangle,
$$

$$
\phi_{i}(r)=\left\langle r\left|a_{i}^{\dagger}\right| \mathrm{vac}\right\rangle
$$

where the single index $i$ represents the complete set of HO quantum numbers $\left(n_{i}, l_{i}, m_{i}\right)$, and where $i=0$ corresponds to the 0 s ground state, $\phi_{0}(r)$. It is important to realize that the emphasis and development of our current approach hereafter diverges from the standard ( $\mathrm{CC}$ ) methodology, and we accordingly do not discuss the latter approach in any further detail.

We have shown elsewhere $[1,6]$ that the most general admixture of $1 \mathrm{p}-1 \mathrm{~h}$ and $2 \mathrm{p}-2 \mathrm{~h}$ excitations with respect to the uncorrelated $(0 \mathrm{~s})^{4}$ configuration, which is both rotationally and translationally invariant (i.e. which leaves the centre of mass in the 
same 0s state as the uncorrelated state $\left.\left|\Phi_{0}\right\rangle\right)$ is generated by the further drastic restriction of the general form of equation (2) to the particular combination,

$$
\hat{S}_{2}=\sum_{n=1}^{\infty} S_{n} \sum_{n_{1} n_{2} l}\left\langle n 0000 \mid n_{1} l n_{2} l 0\right\rangle\left[a_{n_{1} l}^{\dagger} \times a_{n_{2} l}^{\dagger}\right]^{0} a_{0} a_{0} .
$$

The quantity in angular brackets is a Brody-Moshinsky transformation coefficient, and the square bracket indicates the usual coupling to zero total angular momentum of the excited pair. The amplitudes $\left\{S_{n}\right\}$ in equation (5) now therefore correspond to two-particle excitations with energy $2 n \hbar \omega$ relative to the uncorrelated state $\left|\Phi_{0}\right\rangle$. We note too that the sums in equation (5) on the (restricted) single-particle HO quantum numbers $\left\{n_{1}, l_{1}=l\right\}$ and $\left\{n_{2}, l_{2}=l\right\}$ must also include the cases in which either one or the other of the two ostensibly excited nucleons is actually unexcited, corresponding to the terms with $\left(n_{i}=0, l=0\right)$, where $i=1,2$. It is only in this way that the specific operator of equation (5) preserves translational and rotational invariance. It clearly comprises an admixture of both $1 \mathrm{p}-1 \mathrm{~h}$ and $2 \mathrm{p}-2 \mathrm{~h}$ excitations.

Much of our earlier work $[1,6]$ on the translationally- and rotationally-invariant (1p-1h and) $2 \mathrm{p}-2 \mathrm{~h}$ excitations induced by the operator $\hat{S}_{2}$ of equation (5) was actually performed directly in the coordinate-space representation of this но Fock-space operator. This may fairly readily be shown to be given as

$$
\left\langle\boldsymbol{r}_{1} \boldsymbol{r}_{2} \boldsymbol{r}_{3} \boldsymbol{r}_{4}\left|\hat{S}_{2}\right| \Phi_{0}\right\rangle=2 \sum_{i<j}^{4} S_{2}\left(r_{i j}\right)\left\langle r_{1} r_{2} r_{3} r_{4} \mid \Phi_{0}\right\rangle
$$

where $r_{i j}=\left|\boldsymbol{r}_{i}-\boldsymbol{r}_{j}\right|$, in terms of a cluster correlation function $S_{2}(r)$

$$
S_{2}(r)=\sum_{n=1}^{\infty} S_{n}\left(\frac{2^{n} n !}{(2 n+1) ! !}\right)^{1 / 2} L_{n}^{(1 / 2)}\left(\frac{1}{2} \alpha^{2} r^{2}\right)
$$

where $L_{n}^{(1 / 2)}$ is the usual associated Laguerre polynomial.

However, even though we thus largely dealt in our earlier work with the two-body correlations in terms of the coordinate-space correlation function $S_{2}(r)$, this latter function was still determined in terms of the Ho basis coefficients $\left\{S_{n}\right\}$ via the expansion in equation (7), or via an equivalent direct expansion in powers of $r^{2}$. Our motivation for this earlier approach was to maintain close contact with other generalized shell-model calculations. While this coordinate-space expansion is usually more efficient than working directly with equation (5) via its complete set of overlaps with all possible similarly defined translationally- and rotationally-invariant admixtures of $1 \mathrm{p}-1 \mathrm{~h}$ and $2 \mathrm{p}-2 \mathrm{~h}$ states, we have clearly still not freed ourselves from the fundamental use of the same underlying orthogonal single-particle basis of но wavefunctions, and its underlying extremely slow convergence properties. Thus, in practice the sum over $n$ in equation (7) has to be truncated at some upper index $n_{\max }$, which restricts us to excitation energies up to $2 n_{\max } \hbar \omega$. Although the restrictions implied by the use of equation (5) rather than equation (2) have enabled us to proceed $[1,6]$ to unusually high levels of excitation energy, the convergence problems are still severe, as we have discussed earlier.

The new course taken in the present work now stems from the realization that what is really fundamental in our approach is the necessity to seek a reasonable approximate representation of a rather general two-body correlation function $S_{2}(r)$. From this vantage point we may simply view the earlier shell-model-like calculation $[1,6]$ as being based on one particular representation, namely in terms of the 
orthogonal polynomials related to the Ho basis, as in equation (7). In view of the severe convergence problems encountered, it is clear that this (traditional) choice is not a very efficient one, and we are thus naturally led to consider alternatives.

As before, the method that we now present is variational. The particular representation employed draws on a rather old technique first used in molecular physics [11-13], and nowadays known (at least in quantum chemistry) as the method of Gaussian geminal expansion. As previously, we start from a trial four-body state involving up to $2 \mathrm{p}-2 \mathrm{~h}$ excitations of the form,

$$
\begin{aligned}
\Phi\left(r_{1} r_{2} r_{3} r_{4}\right) & \equiv\left\langle r_{1} r_{2} r_{3} r_{4}\left|\left(1+\hat{S}_{2}\right)\right| \Phi_{0}\right\rangle \\
& =\sum_{i<j=1}^{4} f_{2}\left(r_{i j}\right)\left\langle r_{1} r_{2} r_{3} r_{4} \mid \Phi_{0}\right\rangle,
\end{aligned}
$$

where we note that the new correlation function, $f_{2}(r) \equiv \frac{1}{6}+2 S_{2}(r)$, includes both the (constant) uncorrelated component and the (1p-1h and) $2 \mathrm{p}-2 \mathrm{~h}$ translationally- and rotationally-invariant component, $S_{2}(r)$. We now expand the function $f_{2}(r)$ in the overcomplete and non-orthogonal basis of Gaussian wavefunctions, to achieve the approximate representation,

$$
f_{2}(r)=\sum_{n=1}^{N_{\max }} A_{n} \exp \left(-b_{n} r^{2}\right)
$$

where we have indicated that, as in any such practical calculation, the sum must be truncated. An approximate ground-state energy, but which is as before an upper bound on the exact value, is now determined by minimization of the expectation value of the four-nucleon Hamiltonian with respect to the trial states of equations (8) and (9). This is completely equivalent to solving a generalized eigenvalue problem for the linear coefficients $\left\{A_{n}\right\}$ but with the matrix elements being non-linear functions of both the Gaussian parameters $\left\{b_{n}\right\}$ and the Ho scale parameter $\alpha$ involved in $\left|\Phi_{0}\right\rangle$. These latter parameters are determined by some suitable variational search method. We also note that the parameters $\left\{b_{n}\right\}$ may include negative values, so long as the trial wavefunction of equation (8) remains normalizable. In practice, the inclusion of such negative values improves the incorrect long-range behaviour of the uncorrelated wavefunction $\left|\Phi_{0}\right\rangle$ compared with its exact counterpart, and has proved to be important in obtaining good energy values.

In table 1 we present results for the ground-state energy of the ${ }^{4} \mathrm{He}$ nucleus using

Table 1. Results for the ground-state energy in $\mathrm{MeV}$ of the ${ }^{4} \mathrm{He}$ nucleus for several potentials calculated via the various coordinate-space independent-cluster approximations $\left(S_{2} / \mathrm{HOB}, f_{2} / \mathrm{GGB}\right.$ and $\left.f_{2}+f_{3}^{\mathcal{S}}\right)$ discussed in the text. Also shown are results from the uncorrelated independent-particle harmonic oscillator optimization $\left((0 s)^{4}\right)$ and the diffusion Monte Carlo (DMC) calculations of $[1,6,14]$.

\begin{tabular}{lllll}
\hline & \multicolumn{4}{c}{ Potential } \\
\cline { 2 - 5 } Method & B1 & V7 & S3 & MT-V \\
\hline (0s) & -28.2 & -27.3 & -5.9 & -6.4 \\
$S_{2} /$ HOB & -37.80 & -28.62 & -25.34 & -26.77 \\
$f_{2} /$ GGB & -37.82 & -28.66 & -25.37 & -29.40 \\
$f_{2}+f_{3}^{\mathrm{S}}$ & -38.08 & -28.68 & -26.67 & -30.71 \\
DMC & $-38.5 \pm 0.1$ & $-28.6 \pm 0.1$ & $-27.31 \pm 0.05$ & $-31.4 \pm 0.05$ \\
\hline
\end{tabular}


the four Wigner potentials, B1, V7, S3 and MT-V, already discussed above. The first row, labelled $(0 \mathrm{~s})^{4}$, corresponds to a variational minimization with respect to the single parameter $\alpha$ with a trial wavefunction of the uncorrelated $(0 s)^{4}$ form of $\left|\Phi_{0}\right\rangle$ given in equation (1). The second and third rows, labelled $S_{2} /$ HOB and $f_{2} /$ GGB respectively, both represent different approximate attempts at the variational optimization of the energy with respect to the trial wavefunction of the translationally- and rotationally-invariant form of equation (8) discussed above. In the former case, $S_{2} /$ HOB refers to the fact that the two-body correlation $f_{2}(r)$ is first written as $f_{2}(r)=\frac{1}{6}+2 S_{2}(r)$, and $S_{2}(r)$ is then parametrized in the harmonic oscillator basis (HOB) as in equation (6) or (7), with some upper cutoff $n_{\max }$ to the sum on $n$. The corresponding $S_{2} /$ HOB results are then obtained by a variational minimization with respect to the parameters $\left\{\alpha, S_{n} ; n=1,2, \ldots, n_{\max }\right\}$ for the largest value of $n_{\max }$ that was compatible with convergence or that we could otherwise reasonably obtain (and see below). This $S_{2} /$ НОв scheme is precisely what was referred to as the TICI2 method in [1].

By contrast, the $f_{2} /$ GGB results in the third row of table 1 now refer to our new optimization of the correlation function $f_{2}(r)$ in equation (8), carried out in the Gaussian geminal basis (GGB) of equation (9), i.e. with respect to the parameters $\left\{\alpha, A_{n}, b_{n} ; n=1,2, \ldots, N_{\max }\right\}$. There is no doubt that the new results for the ground-state energies are converged to within at least $0.01 \mathrm{MeV}$ to the true minimum for this class of trial wavefunctions, and yet they require values of $N_{\max }$ no greater than seven to achieve this accuracy.

This much more rapid convergence, and the consequent difference in computer time and effort of the $f_{2} / \mathrm{GGB}$ results compared with their $S_{2} /$ HOB counterparts, is impressive. For example, for the $S 3$ potential it was necessary to use a value of $n_{\max }=33$ to achieve the $S_{2} /$ HOB results quoted. This required a CPU time per calculation (i.e. for each value of $\alpha$ ) of about $15 \mathrm{~h}$ on a DEC $\mu$ Vax machine, in a calculation which required quadruple precision to avoid numerical instabilities. (The corresponding $S_{2}$ / HOB results for the $V 7$ and B1 potentials shown in table 1 were performed with values of $n_{\max }=15$ and 30 , respectively.) By contrast, the corresponding $f_{2} /$ GGB results shown took only a few minutes of CPU time on the same machine. Furthermore, this latter time now includes that taken for the complete minimization. Each evaluation of the energy took only a few seconds.

The corresponding difference for the MT-V potential is even more dramatic, since in this case the potential is given as a sum of Yukawa terms and we could not exploit the simple algebraic properties used with the other potentials, which are expressed as sums of Gaussian terms. Thus, for reasons explained in [6], for the MT-V potential we needed to work directly with the Brody-Moshinksy sums implied by equation (5), in order to reach values of $n_{\max }$ as high as 29 , which is the particular result displayed in table 1 . The corresponding calculation in this case was performed on an Amdahl VP1100 vector processor. For each value of the HO parameter $\alpha$, the calculation took $6 \mathrm{~h}$ of CPU time (along with 30 Mbytes of real memory), by far the major part of which was needed to deal with the millions of Brody-Moshinsky brackets required.

The values in the last row of table 1 were obtained by us $[1,6,14]$, using the diffusion Monte Carlo (DMC) method. They may be considered to be exact to within the statistical errors quoted. It is clear that while our $f_{2} /$ GGB results are very good by comparison with the DMC counterparts, there is still a small amount of missing energy in each case, except for the simplest potential, namely V7. Furthermore, 
due to the completely converged nature of our $f_{2} / \mathrm{GGB}$ results, we may now confidently assert that this missing energy is wholly due to the neglect of three- and four-body correlations. We see that these terms must account for about $0.7 \mathrm{MeV}$ binding energy in the case of the effective potential $\mathrm{B} 1$ and about $2 \mathrm{MeV}$ binding for the quasirealistic microscopic potentials S3 and MT-V.

In view of the extreme simplicity of the $f_{2} /$ GGB calculations, it is now possible to contemplate the inclusion of some of these missing $3 p-3 h$ and $4 p-4 h$ configurations. In the first place, it is trivial to see that the magnitudes of the three relative coordinates of a three-body cluster provide a complete translationally- and rotationally-invariant description of it. Thus, the $\mathrm{CM}$ motion is again undisturbed if $3 \mathrm{p}-3 \mathrm{~h}$ correlations are introduced via a symmetric coordinate-space function $f_{3}\left(r, r^{\prime}, r^{\prime \prime}\right)$, in terms of an enlarged trial function of the form

$$
\Phi\left(r_{1} r_{2} r_{3} r_{4}\right)=\left[\sum_{i<j=1}^{4} f_{2}\left(r_{i j}\right)+\sum_{i<j<k=1}^{4} f_{3}\left(r_{i j}, r_{i k}, r_{j k}\right)\right]\left\langle r_{1} r_{2} r_{3} r_{4} \mid \Phi_{0}\right\rangle .
$$

We briefly pause here to discuss a technical point which elucidates the meaning of the term 'independent clusters' used in the title. Thus, in the standard cc calculations the pure two-particle and pure three-particle correlations are treated separately by the cluster operators $\hat{S}_{2}$ and $\hat{S}_{3}$ respectively. In our new formulation, just as the function $f_{2}(r)$ incorporates not only the uncorrelated term but also both the one- and two-body excitations which are handled separately in the cc method by the operators $\hat{S}_{1}$ and $\hat{S}_{2}$ respectively, so the terms involving $f_{2}$ in equation (10) can in practice be dropped as being redundant, since they are in principle subsumed in the remaining $f_{3}$ terms. Thus, for example, the $2 \mathrm{p}-2 \mathrm{~h}$ (and lower-order) excitations expressed by the sum, $f_{2}\left(r_{12}\right)+f_{2}\left(r_{13}\right)+f_{2}\left(r_{23}\right)$, are merely a subset of those $3 \mathrm{p}-3 \mathrm{~h}$ excitations produced by the full three-particle correlation function $f_{3}\left(r_{12}, r_{13}, r_{23}\right)$. If we thus explicitly eliminate the $f_{2}$ terms in equation (10) nothing is lost, and the remaining $f_{3}$ functions are 'independent' in the sense that they are not required either to produce distinct sets of pure $3 \mathrm{p}-3 \mathrm{~h}$ excitations, or separately to reproduce or to build upon earlier sets of lower-order excitations.

Although it is certainly now practicable to implement a full triple Gaussian expansion for the three-body correlation function $f_{3}\left(r^{\prime}, r^{\prime \prime}, r^{\prime \prime \prime}\right)$, comparable with the single Gaussian expansion of equation (9), for the sake of simplicity we first use the much simpler form,

$$
f_{3}\left(r^{\prime}, r^{\prime \prime}, r^{\prime \prime \prime}\right) \rightarrow f_{3}^{S}(r)=\sum_{m=1}^{M_{\max }} C_{m} \exp \left(-d_{m} r^{2}\right)
$$

in terms of the single variable $r \equiv\left(r^{\prime 2}+r^{\prime \prime 2}+r^{\prime \prime \prime 2}\right)^{1 / 2}$, the hyperspherical radius [15] of the three-body subsystem. (We note that in this approximated form, we do now need specifically to retain the $f_{2}$ terms in equation (10), despite our previous remarks.) We expect that the functional form of equation (11) should well describe the $3 p-3 h$ correlations related to the overall size of the excited three-nucleon cluster. The corresponding variational calculation with a trial wavefunction of the form of equations (9)-(11) is scarcely more difficult to implement than the earlier $f_{2} /$ GGB calculation. The results, denoted as $f_{2}+f_{3}^{\mathbf{S}}$, are also shown in table 1 . In view of the extreme simplicity of equation (11), the gain in binding energy is impressive. By comparison with the DMC results, we see that at most $0.7 \mathrm{MeV}$ binding energy is left for the neglected correlations to account for. 


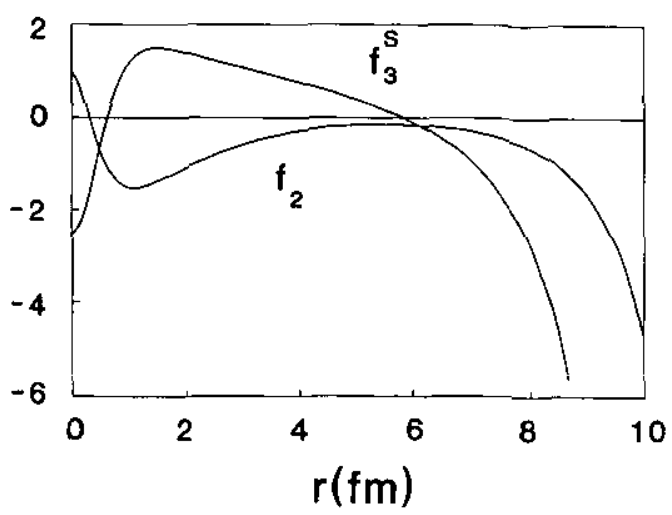

Figure 1. Behaviour of the two correlation functions $f_{2}(r)$ and $f_{3}^{\mathrm{S}}(r)$ described in the text, in the case of the MT-V potential, and using optimized Gaussian expansions with $N_{\max }=M_{\max }=8$.

The actual optimized correlation functions $f_{2}(r)$ and $f_{3}^{\mathrm{S}}(r)$ obtained for the case of the MT-V potential, are displayed in figure 1 for a calculation in which $N_{\max }=M_{\max }=8$, sufficient to achieve an estimate for the ground-state energy which has converged to an accuracy of within at least $0.01 \mathrm{MeV}$ from the true minimum attainable for this $f_{2}+f_{3}^{\mathrm{S}}$ class of trial wavefunctions. The wavefunction is normalized so that $f_{2}(0)=1$. We note first that with our chosen normalization both functions $f_{2}(r)$ and $f_{3}^{\mathrm{s}}(r) \rightarrow-\infty$ as $r \rightarrow \infty$. This behaviour is related to our previous observation that the uncorrelated $(0 \mathrm{~s})^{4}$ HO wavefunction falls off much too rapidly with respect to the true wavefunction as any one of the four nucleons becomes far removed from the remainder. This incorrect long-distance behaviour is partially compensated for by the optimization search, which permits negative values for some members of both sets of Gaussian scale parameters $\left\{b_{n}\right\}$ and $\left\{d_{m}\right\}$, consistent with the overall trial wavefunction remaining bound (i.e. normalizable).

We also note that the long-range behaviours of our correlation functions are very different from that of their comparable counterparts in the Jastrow variational method $[1,14]$. Thus, the Jastrow correlation functions are usually required to approach a constant value as $r \rightarrow \infty$, and their ability to reproduce accurately the very long-range correlations in the nucleus is thereby restricted. (It should be mentioned, however, that this is not their purpose.) It is clear, furthermore, that the Gaussian expansions employed by us are capable, even with relatively few terms, of producing very non-Gaussian-like functions. An example is the almost linear behaviour observed in figure 1 for $f_{3}^{\mathrm{S}}(r)$ for values of $r$ between about $2 \mathrm{fm}$ and $4 \mathrm{fm}$. Finally, it is also interesting to note the way that the behaviour of $f_{3}^{\mathrm{s}}(r)$ tends to oppose that of $f_{2}(r)$ at short and intermediate distances. The three-body correlations thus in some sense counterbalance the two-body correlations at these separations.

In conclusion, we have shown how, in the simple systems considered here, all (one- and) two-particle excitations which leave the $\mathrm{cm}$ in the 0 s state can be generated via the use in coordinate space of a function depending on only one scalar variable, and that this function can be determined optimally and easily via a straightforward variational calculation of the energy after an expansion in the overcomplete basis of Gaussian functions. We may thereby avoid the very poor convergence properties of the equivalent shell-model calculation, in which the 
comparable expansion is in terms of the Ho wavefunctions. We were thus able to obtain very good and rapidly convergent values for the ground-state energy for all potentials considered, and at a very much reduced computational effort. We have also shown how a very simple ansatz for the rather more complicated function describing the three-particle excitations allowed us to obtain most of the missing binding energy. A complete description of these systems would involve the inclusion of the full three-particle function $f_{3}\left(r_{i j}, r_{i k}, r_{j k}\right)$, as well as a corresponding fourparticle excitation function $f_{4}$. We plan to continue with this approach to include all of the three-particle excitations, and at least some of the four-particle excitations. We are also currently working on both the extension of this method to statedependent potentials, which will involve a proper fermionic description of the system, as well as applications to heavier nuclei.

\section{Acknowledgment}

EB and RG acknowledge support from the Comision Interministerial de Ciencia y Tecnología, Spain under contract 969/87. MFF acknowledges support via a fellowship from the Ministerio de Educacion y Ciencia, Spain under the program Estancias Temporales de Cientificos y Tecnologos Extranjeros. RFB acknowledges the support of a research grant from the SERC of Great Britain.

\section{References}

[1] Bishop R F, Flynn M F, Boscá M C, Buendía E and Guardiola R 1990 J. Phys. G: Nucl. Part. Phys. 16 L61

[2] Brink D M and Boeker E 1967 Nucl. Phys. A 911

[3] Volkov A B 1965 Nucl. Phys. A 7433

[4] Ceuleneer R and Vandepeutte P 1985 Phys. Rev. C 311528

[5] Ceuleneer R, Vandepeutte $P$ and Semay C 1987 Phys. Lett. 196B 303

[6] Bishop R F, Flynn M F, Boscá M C, Buendía E and Guardiola R 1990 Phys. Rev. C 421341

[7] Afnan I R and Tang Y C 1968 Phys. Rev. 1751337

[8] Malfliet R A and Tjon J A 1969 Nucl. Phys. A 127161

[9] Kümmel H, Lührmann K H and Zabolitzky J G 1978 Phys. Rep. 36 C1

[10] Bishop R F and Kümmel H G 1987 Phys. Today 40352

[11] Boys S F 1960 Proc. R. Soc. A 258402

[12] Singer K 1960 Proc. R. Soc. A 258412

[13] Longstaff J V and Singer K 1960 Proc. R. Soc. A 258421

[14] Bishop R F, Flynn M F, Boscá M C, Buendía E and Guardiola R 1990 The Nuclear Equation of State, Part A: Discovery of Nuclear Shock Waves and EOS ed W Greiner and H Stöcker (New York: Plenum) p 605

[15] Fabre de la Ripelle M, Fiedeldey H and Sofianos S A 1988 Phys. Rev. C 38449 